\title{
An UPLC-MS/MS method for highly sensitive high-throughput analysis of phytohormones in plant tissues
}

Gerd Ulrich Balcke ${ }^{1 \dagger}$, Vinzenz Handrick ${ }^{1,2+}$, Nick Bergau', Mandy Fichtner ${ }^{1}$, Anja Henning ${ }^{1}$, Hagen Stellmach', Alain Tissier ${ }^{1}$, Bettina Hause ${ }^{1}$ and Andrej Frolov ${ }^{1,3^{*}}$

\begin{abstract}
Background: Phytohormones are the key metabolites participating in the regulation of multiple functions of plant organism. Among them, jasmonates, as well as abscisic and salicylic acids are responsible for triggering and modulating plant reactions targeted against pathogens and herbivores, as well as resistance to abiotic stress (drought, UV-irradiation and mechanical wounding). These factors induce dramatic changes in phytohormone biosynthesis and transport leading to rapid local and systemic stress responses. Understanding of underlying mechanisms is of principle interest for scientists working in various areas of plant biology. However, highly sensitive, precise and high-throughput methods for quantification of these phytohormones in small samples of plant tissues are still missing.
\end{abstract}

Results: Here we present an LC-MS/MS method for fast and highly sensitive determination of jasmonates, abscisic and salicylic acids. A single-step sample preparation procedure based on mixed-mode solid phase extraction was efficiently combined with essential improvements in mobile phase composition yielding higher efficiency of chromatographic separation and MS-sensitivity. This strategy resulted in dramatic increase in overall sensitivity, allowing successful determination of phytohormones in small (less than $50 \mathrm{mg}$ of fresh weight) tissue samples. The method was completely validated in terms of analyte recovery, sensitivity, linearity and precision. Additionally, it was cross-validated with a well-established GC-MS-based procedure and its applicability to a variety of plant species and organs was verified.

Conclusion: The method can be applied for the analyses of target phytohormones in small tissue samples obtained from any plant species and/or plant part relying on any commercially available (even less sensitive) tandem mass spectrometry instrumentation.

Keywords: Phytohormones, Jasmonates, LC-MS/MS, Solid phase extraction (SPE), Quantification, Electrospray ionization, Plant stress response, Wounding

\section{Background}

Plant hormones play a key role in the regulation of plant development and response to various biotic and abiotic stresses. Salicylic acid (SA), abscisic acid (ABA), jasmonic acid (JA) and related compounds (Figure 1) are known to

\footnotetext{
* Correspondence: andrej.frolov@bbz.uni-leipzig.de

${ }^{\dagger}$ Equal contributors

'Department of Cell and Metabolic Biology, Leibniz Institute of Plant Biochemistry, Weinberg 3, Halle (Saale) 06120, Germany

${ }^{3}$ Faculty of Chemistry and Mineralogy, Institute of Bioanalytical Chemistry, Centre for Biotechnology and Biomedicine, Leipzig University, Deutscher Platz 5, Leipzig 04103, Germany

Full list of author information is available at the end of the article
}

participate in plant response against pathogens, herbivores and abiotic factors such as UV irradiation, ozone exposure, high and low temperature, osmotic stress and mechanical wounding [1-5].

Wounding is known to trigger multiple plant defense mechanisms, such as synthesis of proteinase inhibitors, phytoalexins and volatile attractants, all directly mediated by jasmonates $[4,6]$ and influenced by SA [1] and ABA [3]. (+)-7-iso-JA is synthesized in chloroplasts and peroxisomes [5] and is then converted into the biologically active conjugate (+)-7-iso-JA-isoleucine (JA-Ile) [2]. In Arabidopsis thaliana, JA accumulates locally in leaves already

\section{Biomed Central}


(1)

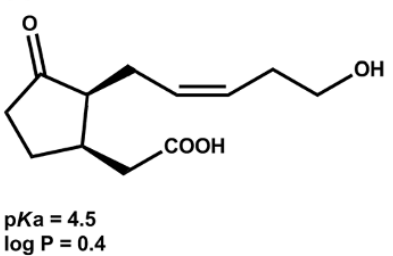

(2)

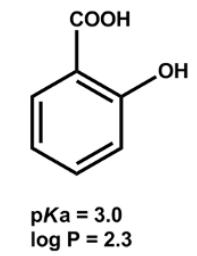

(3)

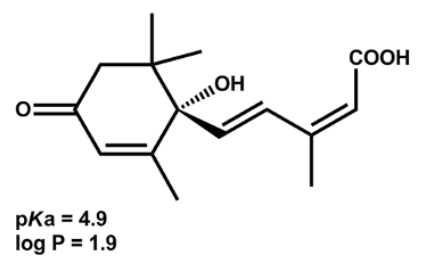

(4)

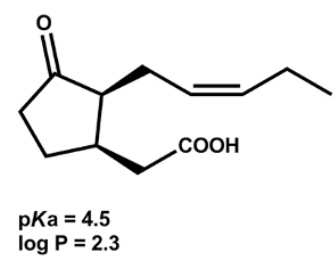

(5)

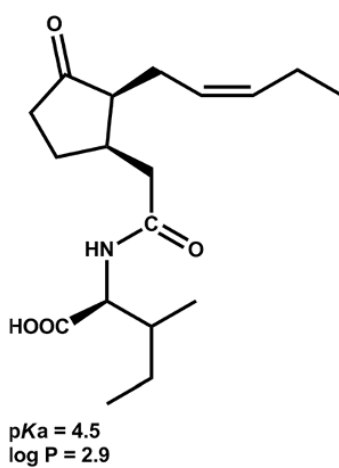

(6)

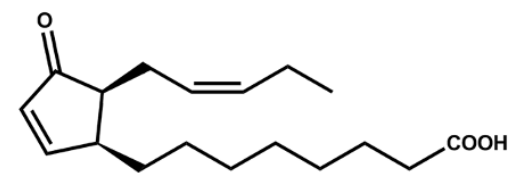

Figure 1 The structures of target phytohormones: 12-hydroxyjasmonic acid, 12-OH-JA- (1); salicylic acid, SA (2); abscisic acid, ABA (3); (+)-7-iso-jasmonic acid, JA (4); (+)-7-iso-jasmonoyl isoleucine, JA-lle (5); cis-12-oxophytodienoic acid, OPDA (6).

$30 \mathrm{~s}$ after wounding, and less than 5 min are necessary for a significant increase in JA-Ile levels [7]. The rise in JA and JA-Ile is accompanied by a rise of cis-12-oxophytodienoic acid (OPDA) in local and systemic leaves, respectively [8]. However, the fine mechanisms underlying distribution of JA, JA-Ile and their precursor OPDA as well as their hydroxylated and carboxylated derivatives [9] within plant tissues are still unknown. To fill this gap, highly-sensitive analytical approaches providing hormone content information for small $(\leq 50 \mathrm{mg}$ ) tissue amounts are required.

Gas chromatography (GC) coupled on-line to mass spectrometry (MS) is routinely applied for the simultaneous analysis of multiple hormones in plant tissues [10]. However, as high analyte volatility is the prerequisite for proper GC-separation, derivatization of hydrophilic groups is required. Besides this relatively time-consuming step, even the modern GC-MS approaches still require at least $300 \mathrm{mg}$ fresh plant material to reach dynamic range of a mass spectrometer [11]. Moreover, in order to reduce signal interference in quadrupole (Q) and ion trap (IT) mass analyzers, additional (most often multistep) depletion of non-hormone compounds is usually required [12]. These two factors dramatically reduce method sensitivity and sample throughput.

Reversed phase-high performance liquid chromatography (RP-HPLC)-electrospray ionization (ESI)-MS is an elegant way to overcome these limitations. High separation efficiency and selectivity of RP-HPLC minimizes ESI matrix effects [13], ensuring sensitive detection of analytes without additional derivatization procedures [9]. Tandem mass spectrometry (MS/MS) with triple quadrupole (QqQ) instruments operating in multiple reaction monitoring (MRM) mode is characterized with favorable duty cycles and reduced chemical noise that provides further sensitivity improvement [14]. Relatively short dwell times ( $\leq 50 \mathrm{~ms}$ ) typical for modern QqQ mass spectrometers make a rapid analysis of multiple hormones possible [15]. Application of ultra performance liquid chromatography (UPLC) [16] as well as solid-phase extraction (SPE)-based enrichment [17] may further improve sensitivity of phytohormone analysis. Nevertheless, obtained in the presence of biological matrix method quantification limits $\left(\mathrm{LOQ}_{\mathrm{m}}\right)$ reported for JA, SA and ABA typically do not exceed $2.5 \times 10^{-14}$ mol (even with top-sensitive instruments). Hence, relatively large sample amounts are still required for analysis.

Here we present a new sensitive, precise and highthroughput UPLC-ESI-MS/MS-based method for simultaneous analysis of jasmonates, $\mathrm{SA}$, and $\mathrm{ABA}$ in plant tissues. After introduction of an SPE-based enrichment/ depletion procedure and adjustment of both chromatographic and mass spectrometric parameters, phytohormones could be detected in plant extracts with LOQs of $10^{-17}-10^{-15} \mathrm{~mol}$. The procedure proved to be fast, universal, reproducible, and provided a throughput of at least 96 samples per day. Finally, the method was 
successfully applied to the study of wound-induced rise in jasmonates in tomato leaves (Solanum lycopersicum, cv. MicroTom), cross-validated with a well-established GC-MS procedure [12] and applied to various plant organs and species.

\section{Results and discussion}

Selection of quadrupole mass ranges for MS/MS analysis In the initial step, SA, ABA, OPDA, JA, JA-Ile and 12hydroxy-JA (12-OH-JA) were annotated in chromatograms of authentic standard mixtures by their retention times, $\mathrm{m} / \mathrm{z}$ values of corresponding $[\mathrm{M}-\mathrm{H}]^{-}$ions and characteristic MS/MS fragmentation patterns (Additional file 1: Table S1). On the basis of this information, compound-dependent parameters, namely collision, declustering, collision cell entrance and exit potentials (CE, DP, CEP and CXP, respectively) were adjusted for selected precursor-fragment pairs by syringe pump infusion of $0.5-1.0 \mathrm{mmol} / \mathrm{L}$ solutions of individual authentic standards using Analyst 5.1 software (Additional file 1: Table S1). At least two Q1/Q3 mass range combinations (MRM-transitions) were selected for each compound. Thus, each analyte was characterized by two specific precursor-fragment ion combinations and a characteristic retention time $\left(t_{R}\right)$. For each analyte, quantification relied on the most intense transition (shown bold in Additional file 1: Table S1), while the less intense one was used to confirm the phytohormone assignment. The transitions, selected here for quantification (225/59, 137/93, 263/153, 209/59, 322/130, 291/165 for 12-OH-JA, SA, ABA, JA, JA-Ile and OPDA, respectively) were shown to yield the most abundant MS-signals also in other studies $[14,18,19]$. In order to correct phytohormone content values for effects related to the plant matrix and for losses during sample preparation, for all analytes we employed internal standardization using corresponding stable isotope-labeled counterparts amended to the extraction solvent. For these compounds $\left({ }^{2} \mathrm{H}_{6}-\mathrm{SA},{ }^{2} \mathrm{H}_{6}-\mathrm{ABA},{ }^{2} \mathrm{H}_{6}-\mathrm{JA}\right.$, ${ }^{2} \mathrm{H}_{2}$-JA-Ile and ${ }^{2} \mathrm{H}_{5}$-OPDA), the Q1 and Q3 mass ranges selected for authentic standards were corrected for the presence of deuterium atoms (Additional file 1: Table S1) while the same set of MS parameters (CE, DP, CEP and CXP) was applied for their quantification as for the nonlabeled isotopologues. As no structure-specific internal standard for 12-OH-JA was available, this compound was standardized by ${ }^{2} \mathrm{H}_{6}$-JA.

\section{Optimization of chromatography system}

The choice of an adequate chromatography system is the most important factor influencing sensitivity of LC-MS analysis. As all analytes presented in Figure 1 contain hydrophobic moieties in their structure, we have chosen RP-HPLC for their separation. In order to increase separation efficiency and, hence, sensitivity of chromatographic analysis while minimizing analysis time, we applied a C18 reversed phase column filled with $1.8 \mu \mathrm{m}$ particles and UPLC instrumentation. Special attention was paid to the mobile phase composition (i.e. organic modifier and additives), as it directly influences both LC-separation and MS-detection [20].

For sufficient retention of acids on reversed phase their protonated state is a prerequisite [21], while basic (i.e. deprotonating) additives enhance ionization in an ESI source operating in negative ion mode [22]. All previous eluent selection strategies relied either on acidic (formic and acetic acids) [15,18] or basic (ammonium acetate) [23] additives favoring only separation or ionization, respectively. Notably, some polar carboxylated phytohormones (e.g. SA) are partly dissociated at $\mathrm{pH}$ 4.0. Thus, their complete retention on $\mathrm{C} 18$ reversed phases in presence of ammonium acetate buffers was impaired (Figure 1). We assumed that the use of ammonium formate buffer adjusted with formic acid to $\mathrm{pH} 3.5$ would improve both chromatographic and ionization behavior of acidic phytohormones. Therefore, the effect of $0.3 \mathrm{mmol} / \mathrm{L}$ formic acid $(\mathrm{pH} 2.7), 0.3 \mathrm{mmol} / \mathrm{L}$ ammonium acetate $(\mathrm{pH} 4.0)$ and $0.3 \mathrm{mmol} / \mathrm{L}$ ammonium formate $(\mathrm{pH} 3.5)$ on intensities of $\mathrm{SA}[\mathrm{M}-\mathrm{H}]^{-}$ions was compared. Indeed, $0.3 \mathrm{mmol} / \mathrm{L}$ ammonium formate resulted in highest signal intensities in Q1 scans (Additional file 1: Figure S1). Reducing or increase of buffer ionic strength did not result in further improvements in ionization efficiency (Additional file 1: Figure S2). Thus, overall 10 and $25 \%$ increase in intensity of phytohormone $[\mathrm{M}-\mathrm{H}]^{-}$ions in comparison to typically used formic acid- and ammonium acetate-based systems was achieved.

Further, the effect of three buffer additives on retention and separation of acidic phytohormones on reversed phase was studied with the mixture of $1 \mu \mathrm{mol} / \mathrm{L} \mathrm{SA}, \mathrm{ABA}$ and JA. Although methanol is widely used as organic modifier in RP-HPLC-based phytohormone analysis [15,18,24,25], here we used only acetonitrile, because of its higher elution power and, hence, higher separation efficiency and sensitivity of analysis [26]. Although $0.1 \%$ formic acid ( $\mathrm{pH} 2.7$ ) provided good retention for all analytes, ABA and SA could not be completely separated under selected gradient conditions (Figure 2A). Increase of eluent $\mathrm{pH}$ to 4.0 $(0.3 \mathrm{mmol} / \mathrm{L}$ ammonium acetate buffer $)$ resulted in essential peak broadening and fronting, whereas use of ammonium formate buffer with $\mathrm{pH} 3.5$ yielded optimal chromatographic performance (Figure $2 \mathrm{~B}$ and $\mathrm{C}$, respectively).

\section{Solid phase extraction}

As phytohormones are present in plant tissues in the concentration range of $10^{-9}-10^{-6} \mathrm{~mol} / \mathrm{L}$ [17], strong enrichment is desired for their adequate quantification by mass spectrometry (especially if only small sample amounts are available). Previously, it was achieved by liquid-phase extraction (LPE) with dichloromethane or 


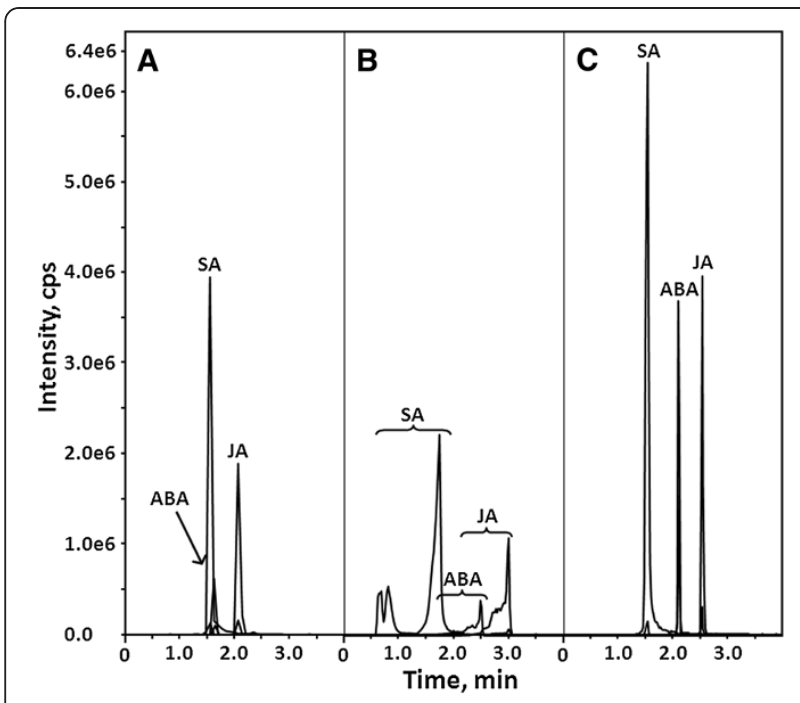

Figure 2 Reversed phase chromatograms of a standard mixture containing $1 \mu \mathrm{mol} / \mathrm{L} \mathrm{SA}, \mathrm{ABA}$ and $\mathrm{JA}$ in $20 \%$ aq. methanol (injection volume of $1 \mu \mathrm{L}$ ) acquired in water-acetonitrile eluent system containing $0.1 \%$ formic acid $(A), 0.3 \mathrm{mmol} / \mathrm{L}$ ammonium acetate (B) or $0.3 \mathrm{mmol} / \mathrm{L}$ ammonium formate (C). The reversed phase chromatograms were recorded by a QqLIT mass spectrometer operating in negative MS/MS (MRM) mode.

diethyl ether $[18,27,28]$ or solid-phase extraction (SPE) using RP $[15,17,25,29]$, anion [30,31] or cation exchange [32] materials. Besides enrichment, these procedures also provide a reduction in sample complexity, minimizing matrix effects during the ESI-process [13]. As most of the published applications rely on C18-RP-SPE, in our initial experiments we used cartridges filled with this type of material (Chromabond C18 ec, Macherey-Nagel, Düren, Germany). However, due to different $\mathrm{pK}_{\mathrm{S}}$ or the $\log \mathrm{P}$ values (Figure 1), the analytes varied strongly in their solubility in aqueous methanolic mixtures and demonstrated different retention behavior on C18 material. Thus, it was impossible to achieve good recovery for all phytohormones in the analyzed standard mixtures (Additional file 1: Figure S3). Even application of 10\% aq. methanol (a concentration of organic modifier necessary for quantitative dissolving of all analytes) as a loading solvent for a mixture of JA, ABA and SA, did not ensure efficient retention of the most hydrophilic component SA. Its loss in the wash fraction was essential (Additional file 1: Figure S3). In contrast, ABA, a more hydrophobic analyte, remained partly retained even after elution with acetonitrile (Additional file 1: Figure S3). Such a behavior of phytohormones on C18 SPE material was not surprising as the restricted applicability of silicabased C18 material for simultaneous enrichment of multiple phytohormones was previously reported [18]. Consequently, SPE procedures based on combination of several (at least two) separation principals, suitable for retention of both hydrophilic and hydrophobic compounds, were expected to provide sufficient retention of all analytes.

In order to select an appropriate phase capable of retaining analytes within a broad range of hydrophobicity and pKa values (Figure 1), a screening experiment with eleven commercially available solid phase materials possessing different chemistry and specificity was performed using a standard mixture of three hormones SA, ABA and JA. To reduce sample preparation time (i.e. to increase sample throughput), we switched to 96well plate scale while performing all SPE steps in a centrifuge at low speed. Among the SPE materials tested we employed three silica-based and two polymer-based reversed phase materials, as well as one polar-functionalized, three anion- and two cation-exchange polymerbased resins (Additional file 1: Table S2). Five ion exchange materials relied on a dual retention mechanism based on hydrophobic interactions with a polymeric backbone in addition to ion exchange on charged surfaces, i.e. so called "mixed mode" phases. To provide a reliable comparison of the analyte recovery from different SPEmaterials, four consecutive elution steps were employed to disrupt hydrophobic, anionic and cationic interactions of analytes with the solid phases (Figure 3A). Flowthrough, wash and four eluate fractions were collected, and $3.3 \mu \mathrm{L}$ of each effluent was subjected to RP-UPLC.

Analysis of the fractions revealed essential breakthrough of all three phytohormones upon sample loading and washing when silica-based C18 reversed phase materials were applied (Figure 3B - D, I - III). Moreover, in contrast to other phases, duration of solvent filtration through cartridge material was irreproducible. It led to lower recovery precision due to drying of the C18 material (Additional file 1: Table S3). As can be seen from Figure $3 \mathrm{~B}-\mathrm{D}(\mathrm{I}-\mathrm{V})$, most of the loaded analyte retained on reversed phase was recovered with acetonitrile in the first elution step (Additional file 1: Figure S4), whereby polymer-based RP materials showed lower losses during loading and washing steps. However, much stronger retention of all analytes on the latter materials (IV - V) and polar-functionalized phase (VI) was observed: even a stronger eluent (isopropanol) did not result in quantitative elution of abscisic acid from polymer-based reversed phases (Figure 3C).

In contrast, all three tested weak anion-exchange resins (VII-IX) as well as the weak cation-exchange resin (XI) provided reduced recoveries for $\mathrm{SA}$ and $\mathrm{ABA}$ (Figure 3B - C). For weak anion exchange resins it could be explained by a strong break-through in flow-through and wash fractions (Figure $3 \mathrm{~B}-\mathrm{C}$ ). In contrast, extremely high retention even for less hydrophobic analytes was observed on the weak cation exchange material (XI): SA, as the strongest acid among the three analytes, resulted in only $24.2 \%$ recovery in all eluate fractions 

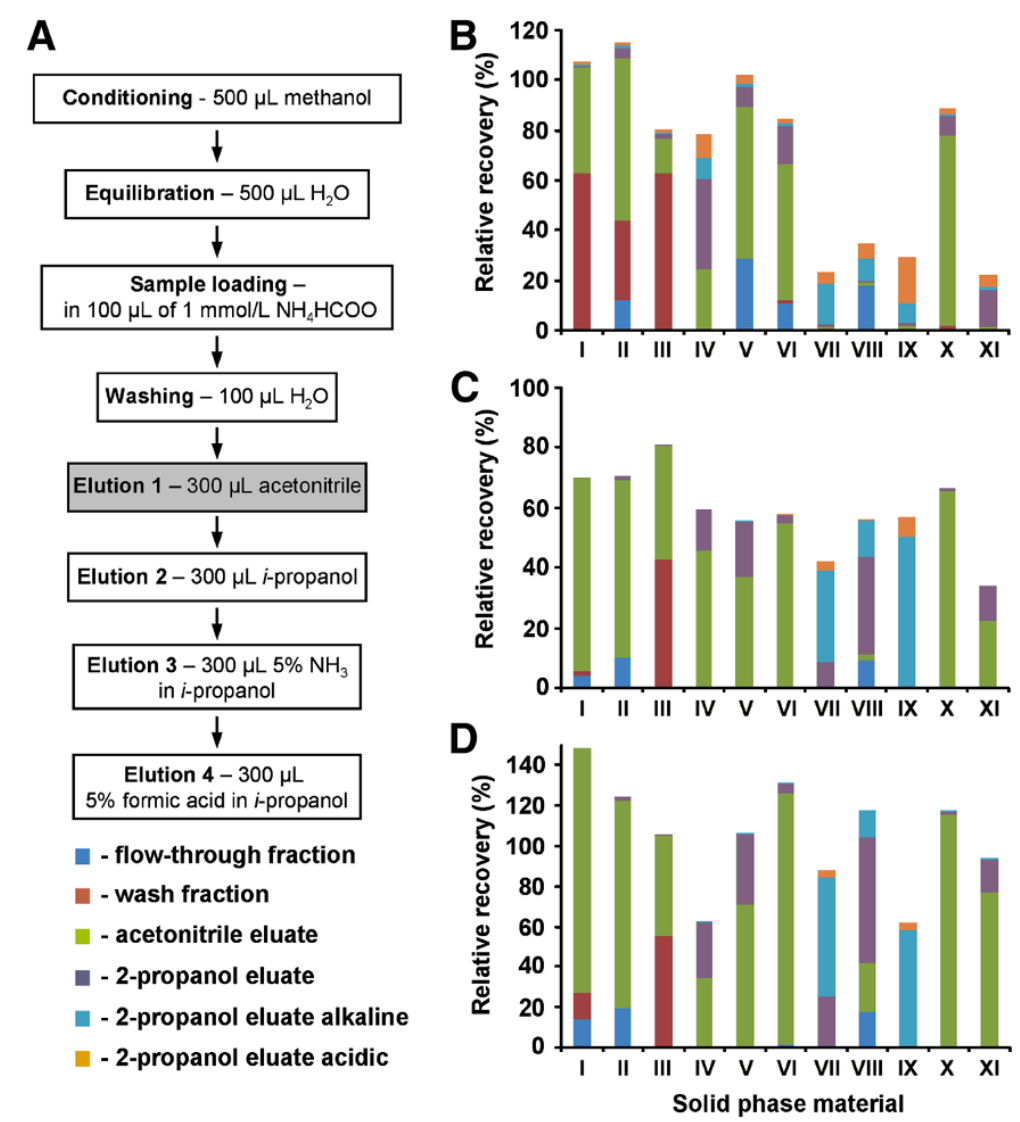

Figure 3 Extraction protocol (A) and recoveries of SA (B), ABA (C) and JA (D) in flow-through (blue), wash (red), acetonitrile elution (green), isopropanol elution (violet), alkaline elution (sky-blue) and acidic elution (orange) fractions observed after loading of a standard mixture containing $19.8 \mathrm{mg} / \mathrm{L}$ of $\mathrm{SA}, \mathrm{ABA}$ and JA $(100 \mu \mathrm{L})$ on solid phase material of different specificity: Chromabond C18ec (I), Strata C18-E (II), Spec C18 AR (III), Chromabond HR-X (IV), OASIS HLB (V), Bond Elut PLEXA (VI), Strata X-AW30u (VII), OASIS WAX (VIII), Chromabond HR-XAW (IX), OASIS MCX (X), OASIS WCX (XI). Measurements were performed in triplicates.

(Figure 3B, Additional file 1: Table S3, ). Most likely, a reversed phase mechanism played essential role in this phenomenon, as elution under alkaline conditions was negligible (Figure 3). It is important to note, that recoveries in acetonitrile fraction were, however, highly reproducible for most of the phases: RSD\% values did not exceed $16 \%$ for any of analytes and were typically less than 10\% (Additional file 1: Table S3).

While relatively good analyte recoveries from weak anion exchange material during alkaline elution is supported by discharge of weak bases at the solid phase surface, high retention of anionic phytohormones on a strong cation exchanger was unexpected. However, due to the dual chemistry of this phase, relatively hydrophobic phytohormones were better retained by a RP mechanism. Additionally, an exchange of cationic metabolites (i.e. counter-ions) present in plant extracts against surface bound protons (H-form) may shift acid-base equilibrium of carboxylated hormones towards the non-dissociated forms, thus enhancing hydrophobic interactions of also relatively polar hormones with polymeric material.
Besides, resulted from ion exchange free protons will only temporarily decrease the $\mathrm{pH}$ since they are washed out upon loading whereas retarded matrix cations are not eluted from the solid phase with base-free acetonitrile. Thereby, depletion of the plant extract for cationic and zwitter-ionic metabolites could be achieved. Possibly, pre-load acidification of hormone extracts would result in better retention of phytohormones on RP materials. However, as a pH decrease might influence hormone stability, use of cation exchange SPE seems to be favorable. Not less important, exchange of matrix cations reduces sample complexity diminishing thereby possible matrix effects during electrospray ionization.

On the basis of the obtained results, strong cation exchange polystyrene/ divinylbenzene (PS/DVB)-resin was selected for further experiments. In order to reduce analysis costs, for further analyses we switched to PS/DVB-based Cromabond $^{\circledR}$ HR-XC material (Macherey-Nagel, Düren, Germany), a close analog to the OASIS MCX phase. High recoveries from this phase were confirmed for all six phytohormones of interest in additional experiments (see below). 


\section{Method validation}

Due to improved mobile phase composition, the LCMS/MS method demonstrated remarkable sensitivity: the instrument limits of detection and quantification $\left(\mathrm{LOD}_{\mathrm{i}}\right.$ and $\mathrm{LOQ}_{\mathrm{i}}$, respectively) were between 25 amol for JA-Ile and $2.5 \mathrm{fmol}$ for 12-OH-JA (Additional file 1: Table S4). That is, to the best of our knowledge, superior in comparison to the most of published methods $[17,18,25,33,34]$. Recently, Liu and collaborators reported similar method sensitivity parameters [35]. However, the authors employed a much more sensitive (and, hence, expensive) mass spectrometer. In our study, all analytes demonstrated high linear dynamic ranges (LDRs) typically more than $1 \times 10^{3}$ (only for 12-OH-JA this value was $4 \times 10^{2}$ ), with $R^{2}>0.99$ (Additional file 1: Table S4). The high sensitivity can be explained by employment of UPLC technique [36], providing superior efficiencies of separation and, hence, typical peak widths $\left(\mathrm{w}_{1 / 2}\right)$ of several seconds. However, due to pronounced matrix effects produced by multiple components of plant extracts, the limits of quantification $\left(\mathrm{LOQ}_{\mathrm{m}}\right)$ were expected to be essentially higher.

To determine the analyte $\mathrm{LOQ}_{\mathrm{m}}$ values in a representative plant matrix, a standard addition approach was employed. For this, methanolic plant extracts obtained from $20 \mathrm{mg}$ of fresh material were spiked with a mixture of stable isotope-labeled phytohormone standards prior to SPE enrichment and LC-MS/MS analysis. Assuming the same analytical response (i.e. the same ionization efficiencies and fragmentation patterns) for these stable isotope-labeled compounds as for their natural counterparts, the $\mathrm{LOQ}_{\mathrm{m}}$ values might correspond well to those for the native plant hormones. All together, eight concentration levels of plant hormones were applied. The $\mathrm{LOQ}_{\mathrm{m}}$ values were determined as amounts or contents (in fmol or $\mathrm{ng} / \mathrm{mg}$, respectively) corresponding to the lowest analyte signal/noise ratios yielding significantly higher response in comparison to a next lower concentration level when tested by the paired t-test at 0.05 error probability [37]. The observed $\mathrm{LOQ}_{m}$ values were in lower femtomole range (Table 1) being superior in comparison to other data obtained with the standard addition method $[7,19]$.

In order to further increase the method sensitivity, the eluate SPE fractions were completely dried and reconstituted in small volumes of solvent. Among three tested approaches, drying under nitrogen flow yielded the highest recoveries while $50 \%$ aq. acetonitrile proved to be the most suitable solvent for sample reconstitution, providing at least $70 \%$ recovery for all analytes (Additional file 1: Table S5). These results were in accordance with the relatively high hydrophobicity of OPDA (Figure 1). This analyte could not be completely dissolved in aqueous solutions with low organic content. Therefore, the dried samples were first reconstituted in acetonitrile and diluted afterwards in a $1: 1(\mathrm{v} / \mathrm{v})$ ratio with $0.3 \mathrm{mmol} / \mathrm{L}$ aq. $\mathrm{NH}_{4} \mathrm{HCOO}$. This procedure provided an additional 5.5 - 10.0-fold reduction of $\mathrm{LOQ}_{\mathrm{m}}$ values (Table 1 ). Thus, the method presented here can be successfully applied to phytohormone quantification in tissue samples of less than $50 \mathrm{mg}$. This reduction of sample amount would provide a possibility for characterization of phytohormone gradients in plant organs, i.e. to get a deeper understanding of the mechanisms of hormone-mediated signaling.

The recoveries of all six phytohormones were determined by a standard addition method. For this, SA, ABA, JA, 12-OH-JA, JA-Ile and OPDA were diluted in methanol to the concentrations of $0,1.3,2.5,5.0$ and $10 \mu \mathrm{g} / \mathrm{L} .500 \mu \mathrm{L}$ of these standard mixtures were used for extraction in presence and absence of complex plant matrix and subjected afterwards to RP-UPLC-MS/MS with and without SPE enrichment (see Methods). The analyte abundances (peak heights) were plotted against their corresponding concentrations and linear regression parameters were derived (Table 2). The phytohormone recoveries were determined as slope ratios obtained from concentration-intensity plots based on the data acquired with and without SPE (Table 2). All analytes showed high recoveries ( $88 \%$ or more) irrespective of the interactions with components of plant extract or matrix effects during electrospray ionization. However,

Table 1 Method limits of quantification $\left(\mathrm{LOQ}_{\mathrm{m}}\right)$ determined in complex plant extracts

\begin{tabular}{ccc}
\hline Stable isotope labeled standards ${ }^{\mathbf{a}}$ & LOQ, nmol/L & LOQ, nmol/L, concentrated sample \\
\hline${ }^{2} \mathrm{H}_{6}-\mathrm{SA}$ & 5.80 & 0.58 \\
\hline${ }^{2} \mathrm{H}_{6}-\mathrm{ABA}$ & 12.93 & 2.34 \\
\hline${ }^{2} \mathrm{H}_{6}-\mathrm{JA}$ & 12.93 & 1.81 \\
\hline${ }^{2} \mathrm{H}_{2}-(-)-J A-l l e$ & 1.73 \\
\hline${ }^{2} \mathrm{H}_{5}$-OPDA & 1.89 \\
\hline
\end{tabular}

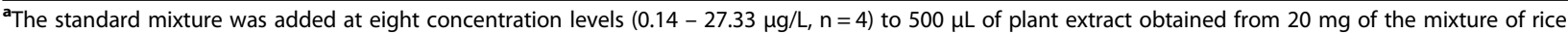
leaf, rice root, tomato leaf, tomato root, potato leaf and Arabidopsis leaf. The spiked extracts were subjected to SPE and LC-MS/MS analysis as described in Methods part with and without subsequent drying/reconstitution step. $3.3 \mu \mathrm{L}$ of SPE eluate was injected in UPLC.

b The SPE eluates were dried completely under nitrogen stream and reconstituted in $40 \mu \mathrm{L}$ acetonitrile. After addition of $40 \mu \mathrm{L} 0.3 \mathrm{mmol} / \mathrm{L}$ aq. ammonium formate, the samples were transferred to glass vials and $3.3 \mu \mathrm{L}$ of solution was injected in UPLC. 
Table 2 Recovery of phytohormones from different sample matrices on polystyrene/ divinylbenzene-based Cromabond $^{\circledR}$ HR-XC material

\begin{tabular}{|c|c|c|c|c|c|c|c|c|c|c|c|c|c|c|c|c|c|c|}
\hline \multirow[t]{2}{*}{ Matrix } & \multicolumn{3}{|c|}{ SA } & \multicolumn{3}{|c|}{ ABA } & \multicolumn{3}{|c|}{ JA } & \multicolumn{3}{|c|}{ 12-OH-JA } & \multicolumn{3}{|c|}{ JA-Ile } & \multicolumn{3}{|c|}{ OPDA } \\
\hline & Slope & $\mathrm{R}^{2}$ & Rec. & Slope & $\mathrm{R}^{2}$ & Rec. & Slope & $\mathrm{R}^{2}$ & Rec. & Slope & $\mathrm{R}^{2}$ & Rec. & Slope & $\mathrm{R}^{2}$ & Rec. & Slope & $\mathrm{R}^{2}$ & Rec. \\
\hline Recovery control & 453.48 & 0.99 & 1.00 & 75.12 & 0.99 & 1.00 & 105.27 & 0.99 & 1.00 & 431.91 & 0.99 & 1.00 & 541.36 & 0.99 & 1.00 & 126.64 & 0.99 & 1.00 \\
\hline 20\% Methanol & 397.59 & 0.99 & 0.88 & 90.73 & 0.99 & 1.21 & 110.37 & 0.97 & 1.05 & 497.68 & 1.00 & 1.15 & 533.24 & 1.00 & 0.98 & 122.33 & 1.00 & 0.97 \\
\hline Methanolic extract & 558.56 & 0.96 & 1.23 & 76.50 & 0.92 & 1.02 & 108.49 & 0.97 & 1.03 & 444.99 & 0.98 & 1.03 & 532.82 & 1.00 & 0.98 & 118.41 & 0.99 & 0.93 \\
\hline $\begin{array}{l}\text { Reconstituted } \\
\text { methanolic extract }\end{array}$ & 392.81 & 0.77 & 0.86 & 55.25 & 0.86 & 0.49 & 81.27 & 0.88 & 0.64 & 156.24 & 0.83 & 0.44 & 424.25 & 0.99 & 0.67 & 77.65 & 0.95 & 0.50 \\
\hline
\end{tabular}

The analyte abundances (peak heights) were plotted against their defined concentrations and correlation of these two parameters was estimated (linear regression coefficient was calculated). The phytohormone recoveries (Rec.) were determined as ratios of slopes compairing corresponding concentration-intensity plots obtained with and without SPE procedure.

as the extracts were further concentrated via a drying/ reconstitution procedure, recovery of all hormones besides SA declined. Moreover, lower $\mathrm{R}^{2}$ values for concentration-analyte intensity plots obtained for dried/ reconstituted standard mixtures indicated distinctly lower precision of this strategy. These losses may be, however, corrected by the use of stable isotope-labeled internal standards. This approach would further decrease the sample amount required for the hormone assessment. However, as desired sensitivity and precision was achieved here without sample drying/reconstitution, this variant of the method was not further investigated.

\section{Study of wounding kinetics in solanum lycopersicum}

In the next step the designed method was verified by recording the levels of jasmonates in mechanically wounded leaves of tomato (Solanum lycopersicum, cv. MicroTom). To elucidate the kinetics of OPDA, JA and JA-Ile, methanolic extracts were obtained in triplicates from leaves at different time points after wounding and were spiked with the mixture of internal standards as described in Methods. The individual extracts were then split in two aliquots, one of which was analyzed by the novel LC-MS/MS method, while the second one was subjected to a well-established GC-MS procedure in a cross-validation experiment [12].

Mechanical wounding is one of the best-elucidated triggers of a local rise in jasmonate levels $[5,6]$. The dynamics of jasmonate contents in wounded tomato leaves is well-characterized. JA levels [31,38,39] increase already within minutes after wounding reaching the maximum $30 \mathrm{~min}$ after stress exposure. This increase is accompanied by accumulation of the physiologically active hormone JA-Ile, the contents of which, however, typically do not exceed the maximal levels detected for JA [9]. Also for the tomato cultivar MicroTom, the data mirror this pattern: the maximum contents of both JA and JA-Ile were detected at $20 \mathrm{~min}$ after wounding (Figure 4A and B). Differences in timing and height of these maxima in the different experiments (data shown in Figure 4 versus [31]) might be due to differences in the tomato cultivar, the developmental stage of plants, the growth conditions and the strength of wounding. As expected, the content of the JA precursor OPDA was slightly increased already $10 \mathrm{~min}$ after wounding (Figure 4C). Most importantly, the results obtained with the new LC-MS/MS method were in agreement with the data obtained from the identical samples using GC-MS This demonstrates the accuracy of JA, JA-Ile and OPDA determination by the new method.

\section{Applicability of the method to other plant species and organs}

In order to check applicability of the analytical approach presented here to other plant species, developmental stages and organs, the method was cross-validated with plant material of different origin: rice (Oryza sativa) roots, Arabidopsis thaliana seedlings and rosette leaves, Medicago truncatula roots and leaves. All the samples obtained all from wild type and non-stressed plants (50 $\mathrm{mg}$ fresh weight, $\mathrm{n}=5$ ) and were extracted as described for tomato leaf, subjected to SPE and the eluates were analyzed for phytohormone contents by RP-UPLC-MS/MS. The resulted values (Additional file 1: Table S6) were in accordance with published data [12,40-42]. Thus, the method was shown to be applicable for any plant object and can be used in any field of plant biology and biochemistry.

In summary, our method delivers reliable values for phytohormone contents in different plant species or plant organs, such as roots, stems, flowers, and seeds. In comparison to existing hormone quantification methods it provides superior sensitivity, precision, and linear dynamic ranges for six plant hormones tested. The resulted concentrations correspond well to those obtained in an independent cross-validation experiment with another analytical approach.

\section{Conclusions}

Jasmonates, ABA and SA are the key players in the regulation of plant defense against pathogen and herbivore attack. Knowledge about their spatial and temporal 


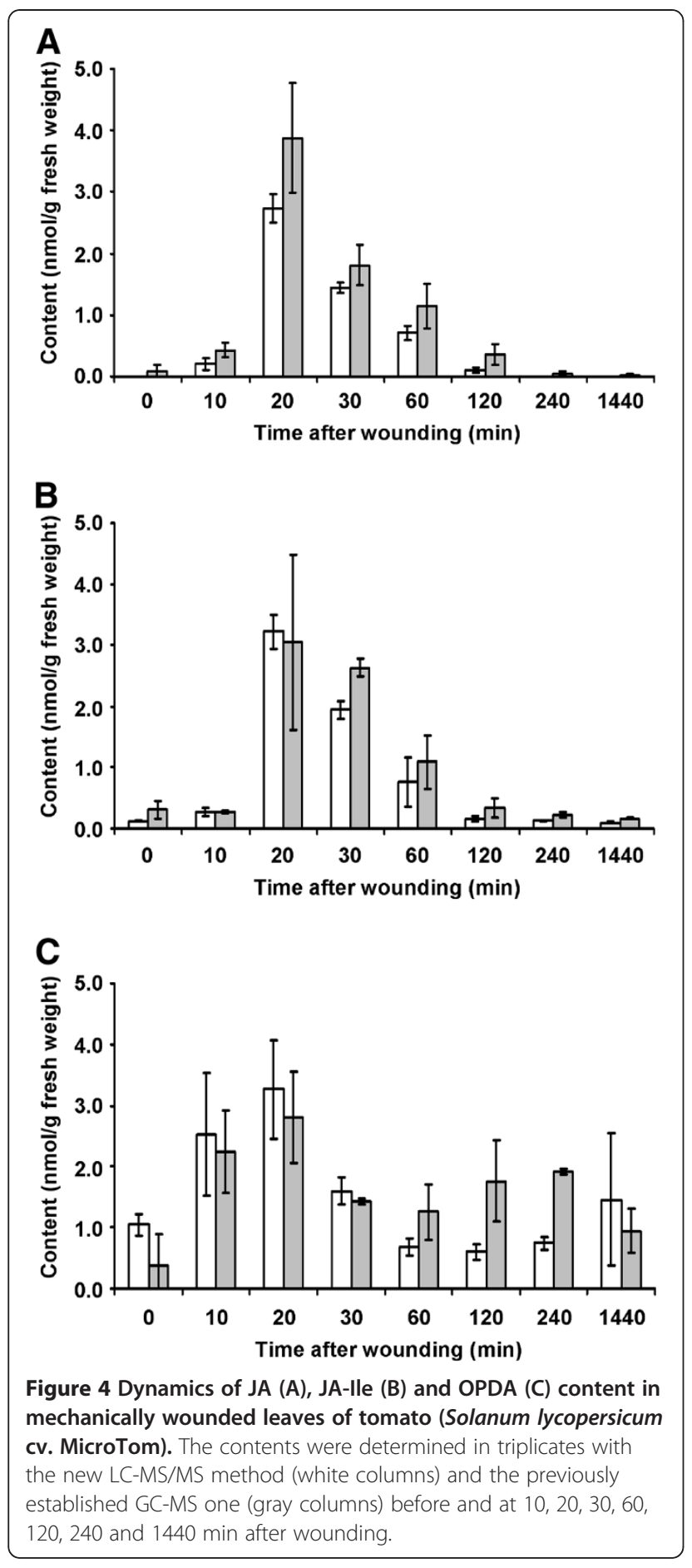

distribution in plant tissues is necessary for understanding the mechanisms of local and systemic plant response to these biotic stress factors. However, for quantification of phytohormones highly sensitive and precise analytical methods are required. We have introduced a highthroughput LC-MS/MS-based approach for absolute quantification of four jasmonates, $\mathrm{SA}$ and $\mathrm{ABA}$ in methanolic extracts of plant tissue. In the sample preparation step, a quantitative enrichment of phytohormones and depletion of cationic metabolites and photosynthetic pigments was achieved on mixed-mode $\mathrm{RP} /$ cation exchange polymeric resins. Besides, LC mobile phase composition was adjusted to provide optimal chromatographic resolution and higher MS-ionization efficiency. Combined, these approaches resulted in a dramatic sensitivity increase in comparison to previously established methods. Due to this sensitivity gain, phytohormone analysis can be successfully performed with a wide range of commercially available mass spectrometers with a low plant material consumption. The validated method was successfully applied to quantitative characterization of jasmonate concentration dynamics in Solanum lycopersicum leaf tissues and crossvalidated with a well-established GC-MS method.

\section{Methods}

\section{Reagents}

$( \pm)$ Abscisic acid (ABA), salicylic acid (SA), ammonium acetate, ammonium formate, formic acid, acetonitrile and methanol (all LC-MS grade) were from Sigma Aldrich (Taufkrichen, Germany). JA was obtained by alkali hydrolysis of methyl jasmonate (MeJa, Firmenich, Geneva, Switzerland). JA-Ile was kindly provided by Dr. Kramell (IPB). 12-Hydroxyjasmonic acid (12-OH-JA) was obtained as described by Kitahara et al. [43]. OPDA was synthesized from linolenic acid using linseed enzyme [44]. ${ }^{2} \mathrm{H}_{6}$-ABA was synthesized according to GómezCadenas et al. [45]. ${ }^{2} \mathrm{H}_{6}$-JA was obtained as described by Miersch et al. [46]. ${ }^{2} \mathrm{H}_{2}-(-)$-JA-Ile was prepared from ${ }^{2} \mathrm{H}_{2}-( \pm)-\mathrm{JA}$ and Ile [47]. ${ }^{2} \mathrm{H}_{5}$-OPDA was prepared from $\left(17-{ }^{2} \mathrm{H}_{2}, 18-{ }^{2} \mathrm{H}_{3}\right)$-linolenic acid as described by Zimmerman and Feng [44]. ${ }^{2} \mathrm{H}_{6}$-SA was purchased from Campro Scientific (Veenendaal, Netherlands). Water was purified in-house by a Milli-Q Plus Ultrapure Water System (Millipore $\mathrm{GmbH}$, Schwalbach, Germany) (resistance $>18 \mathrm{M} \Omega$ ).

\section{Extraction of phytohormones from plant tissues}

$20-50 \mathrm{mg}$ of fresh plant material was homogenized in a mortar under liquid nitrogen and extracted with 500 $\mu \mathrm{L}$ methanol containing $0.1 \mathrm{ng} / \mu \mathrm{L}$ of each stable isotope-labeled internal standard $\left({ }^{2} \mathrm{H}_{6}-\mathrm{SA},{ }^{2} \mathrm{H}_{6}\right.$-ABA, ${ }^{2} \mathrm{H}_{6}$-JA, ${ }^{2} \mathrm{H}_{2}$-(-)-JA-Ile and ${ }^{2} \mathrm{H}_{5}$-OPDA). The extraction was performed in $1.6 \mathrm{~mL}$ cryo-tubes (Precellys Steel Kit $2.8 \mathrm{~mm}$, Peqlab Biotechnologie $\mathrm{GmbH}$, Erlangen, Germany) using a bead mill (FastPrep24 instrument, MP Biomedicals LLC, Santa Ana, CA, USA) with acceleration of $6.5 \mathrm{~m} / \mathrm{s}^{2}$ for $30 \mathrm{~s}$. After centrifugation at $20000 \mathrm{~g}\left(2 \mathrm{~min}, 0^{\circ} \mathrm{C}\right), 450 \mu \mathrm{L}$ of supernatant was transferred into a polypropylene tube, diluted with water to $5 \mathrm{~mL}$ and subjected to solid-phase extraction (SPE). 


\section{Solid-phase extraction (SPE)}

Solid phase extraction was performed in a 96-well plate format using filter plates and deep well receiving plates in conjunction with centrifugation. For the phase screening experiments, 96-deep well filter plates (Agilent Technologies, Böblingen, Germany) were packed with $15-60 \mathrm{mg}$ of SPE materials representing different chemistry and specificity (Additional file 1: Table S2). For the solid phase screening experiment, the plates were conditioned with $500 \mu \mathrm{L}$ methanol and equilibrated with $500 \mu \mathrm{L}$ of water before loading of the standard mixture containing $18.8 \mathrm{mg} / \mathrm{L} \mathrm{SA}, \mathrm{ABA}$ and JA dissolved in $1 \mathrm{mmol} / \mathrm{L}$ ammonium formate. After washing with 100 $\mu \mathrm{L}$ of water, phytohormones were consecutively eluted by $300 \mu \mathrm{L}$ portions of acetonitrile, isopropanol, $5 \%$ aq. $\mathrm{NH}_{4} \mathrm{OH}$ in isopropanol, and 5\% formic acid in isopropanol. All steps were performed by centrifugation at $10 \mathrm{~g}$ (Avanti J-E centrifuge, Beckman-Coulter, Kreefeld, Germany). The fractions were collected in separate tubes and stored at $-20^{\circ} \mathrm{C}$.

For analysis of plant samples and calibration mixtures, the same 96-well filter plates were packed with $50 \mathrm{mg}$ of a strong cation exchange HR-XC material (Macherey \& Nagel, Düren, Germany). The material was conditioned with $1 \mathrm{~mL}$ methanol and equilibrated with $1 \mathrm{~mL}$ water. Afterwards, the plant extracts, diluted as described previously, were loaded on each well in five $1 \mathrm{~mL}$-portions. The fraction containing phytohormones was eluted by $900 \mu \mathrm{L}$ acetonitrile without additional washing. $850 \mu \mathrm{L}$ of the eluate fraction were transferred to a $1.5-\mathrm{mL}$ polypropylene tube and storied at $-20^{\circ} \mathrm{C}$ before LC-MS analysis. Alternatively, eluate fractions were completely dried before storage.

\section{RP-UPLC-ESI-MS/MS}

Separations were performed on a Waters HSS T3 C18 column $(1 \times 100 \mathrm{~mm}$, particle size $1.8 \mu \mathrm{m})$ at $40^{\circ} \mathrm{C}$ using a Waters ACQUITY UPLC System, equipped with an ACQUITY Binary Solvent Manager and ACQUITY Sample Manager (20 $\mu \mathrm{L}$ sample loop, partial loop injection mode, $3.3 \mu \mathrm{L}$ injection volume). Eluents $\mathrm{A}$ and $\mathrm{B}$ were water and $90 \%$ aq. acetonitrile, respectively, both containing $0.1 \% \mathrm{HCOOH}, 0.3 \mathrm{mmol} / \mathrm{L} \mathrm{NH}_{4} \mathrm{CH}_{3} \mathrm{COO}$ (adjusted to $\mathrm{pH} 4.0$ with acetic acid), or $0.3 \mathrm{mmol} / \mathrm{L}$ $\mathrm{NH}_{4} \mathrm{HCOO}$ (adjusted to pH 3.5 with formic acid). Elution was performed isocratically for $0.5 \mathrm{~min}$ at $5 \%$ eluent $\mathrm{B}$ and then consecutive linear gradients to 30,80 and $95 \%$ eluent B in 5, 0.5 and $2 \mathrm{~min}$, respectively were run. The column was re-equilibrated for $3 \mathrm{~min}$. The flow rate was set to $150 \mu \mathrm{L} / \mathrm{min}$ and the column temperature was maintained at $40^{\circ} \mathrm{C}$. Phytohormones were detected online by ESI-MS/MS using a 3200 Q TRAP ${ }^{\circledR}$ LC/MS/MS System hybrid QqLIT mass spectrometer equipped with an ESI-TurboIon-Spray ${ }^{\mathrm{ma}}$ interface, operating in negative ion mode and controlled by Analyst 1.5 software (AB Sciex, Darmstadt, Germany). The LC-ESI source operation parameters were as the following: ion spray voltage, -2700 V; nebulizing gas, 40 psi; source temperature, $550^{\circ} \mathrm{C}$; drying gas, $40 \mathrm{psi}$; curtain gas, $25 \mathrm{psi}$. Instrument tuning and mass calibration were performed with $100 \mu \mathrm{mol} / \mathrm{L}$ polypropylene glycol solutions.

QqQ scans were acquired as multiple reaction monitoring (MRM) experiments with Q1 and Q3 resolution set as "unit". Dwell times were defined by "scheduled MRM" function set as following: MRM detection window, $70 \mathrm{~s}$ and targeted scan time, $1 \mathrm{~s}$. Compounddependent parameters for authentic standards were optimized individually in flow injection experiments and further applied to their stable isotope-labeled counterparts (summarized in Additional file 1: Table S1, ). For these measurements, the ESI source operation parameters were set as following: ion spray voltage, $-4500 \mathrm{~V}$; nebulizing gas, $30 \mathrm{psi}$; source temperature, room temperature (RT); drying gas, 0 psi; curtain gas, 10 psi.

For infusion experiments, a syringe pump (Hamilton OEM Syringe Pump, $10 \mathrm{~mL}$ Syringe, Hamilton Bonaduz AG , Bodanuz, Switzerland) operating at $10 \mu \mathrm{L} / \mathrm{min}$ was coupled on-line to the same ESI-QqLIT mass spectrometer operating in negative ion mode. Individual analytes were dissolved in aq. formic acid ( $\mathrm{pH}$ 2.7), ammonium acetate and ammonium formate buffers (adjusted to $\mathrm{pH}$ 4.0 and 3.5 with acetic and formic acids, respectively) at the concentration of $1 \mu \mathrm{mol} / \mathrm{L}$. The concentrations of solvent additives ranged from 0.1 to $3.0 \mathrm{mmol} / \mathrm{L}$. The analytes were tracked in Q1 scans by their $[\mathrm{M}-\mathrm{H}]^{-}$ions.

\section{Method validation}

For external calibration and instrument detection $\left(\mathrm{LOD}_{\mathrm{i}}\right)$ and quantification $\left(\mathrm{LOQ}_{\mathrm{i}}\right)$ limit determination, the mixture containing $1 \mathrm{mmol} / \mathrm{L}$ of each individual standard was serially diluted with $20 \%$ aq. methanol by $2.0-2.5$ fold increment to obtain 23 concentration steps $(0.01 \mathrm{nmol} / \mathrm{L}-500 \mu \mathrm{mol} / \mathrm{L})$. The method limits of quantification $\left(\mathrm{LOQ}_{\mathrm{m}}\right)$ were determined by the standard addition method [37]. For this, $20 \mathrm{mg}$ of lyophilized plant pool (i.e. a mixture consisting of tomato leaves and roots, potato leaves, rice leaves and roots, barley leaves and Arabidopsis leaves mixed in equal proportion) was spiked with a standard mixture containing ${ }^{2} \mathrm{H}_{6}-\mathrm{SA},{ }^{2} \mathrm{H}_{6}$ ABA, ${ }^{2} \mathrm{H}_{6}$-JA, ${ }^{2} \mathrm{H}_{2}-(-)$-JA-Ile, ${ }^{2} \mathrm{H}_{5}$-OPDA at eight concentration levels $(0.14-27.33 \mu \mathrm{g} / \mathrm{L})$. The spiked samples were extracted with methanol and subjected to SPE as described above.

Recoveries of phytohormones on PS/DVB-based Cromabond $^{\circledR}$ HR-XC material (Macherey \& Nagel, Düren, Germany) were determined by the standard addition method. SA, ABA, JA, 12-OH-JA, JA-Ile and OPDA were diluted in $500 \mu \mathrm{L}$ aliquots of methanol at the 
concentrations of $0,1.3,2.5,5.0,10.0$ and $15 \mu \mathrm{g} / \mathrm{L}$ and processed with and without extraction of complex lyophilized plant pool $(20 \mathrm{mg})$. The obtained extracts were subjected to RP-LC-MS/MS with and without further drying in nitrogen stream. The residues were reconstituted in $40 \mu \mathrm{L}$ acetonitrile before further dilution to $80 \mu \mathrm{l}$ with $0.3 \mathrm{mmol} / \mathrm{L}$ aq. ammonium formate ( $\mathrm{pH} 3.5)$.

The analyte abundances expressed as peak heights were plotted against their concentrations. Additionally, regression coefficients and $R^{2}$ values were calculated.

\section{Plant material and wounding experiments}

Seeds of tomato plants (Solanum lycopersicon cv. MicroTom) were germinated on expanded clay of 2-5 mm particle size (Original Lamstedt Ton; Fibo ExClay, http://www.fiboexclay.de) watered with tape water. After one week, seedlings were transferred into soil, grown in a phytotron at $29^{\circ} \mathrm{C}, 50 \%$ relative humidity and 18 -h light/6-h dark cycle, and were watered every second day with tape water. Oryza sativa L., Arabidopsis thaliana (L.) Heynh and Medicago truncatula Gaertn. plants were grown as described elsewhere [12,40-42]. Wounding of tomato plants was performed at onset of flowering (about six weeks after sowing) and was done by squeezing the leaflets of two leaves per plant five times across the mid-vein with tweezers. For each point a separate plant was used. The tomato leaves (two leaves per plant only) were harvested before wounding and 10, 20, 30, 60, 120, 240 and $1440 \mathrm{~min}$ post-treatment. Tissue extraction, SPE and RP-HPLC-MS/MS were performed as described above. The organs obtained from unstressed plants of other species (O. sativa roots, A. thaliana seedlings and rosette leaf, $M$. truncatula roots and leaf) were treated in the same way. The content of individual phytohormones was calculated as the ratio of analyte and internal standard peak heights multiplied by the concentration of the corresponding internal standard.

\section{Quantitative determinations of OPDA, JA and JA-lle by GC-MS}

Methanolic extracts obtained from wounded tomato leaves were subjected to the well established GC-MS-based strategy comprising ion exchange chromatography and RP-HPLC purification steps [12].

\section{Additional file}

Additional file 1: Supplementary information.

\section{Competing interests}

The authors declare that they have no competing interests

\section{Authors' contributions}

$\mathrm{GB}, \mathrm{VH}, \mathrm{BH}$ and $\mathrm{AF}$ designed the experiments; $\mathrm{AH}$ and $\mathrm{HS}$ performed sample preparation; VH, NB and MF performed UPLC-MS/MS analyses; GB, VH, AT, BH and AF were involved in writing the manuscript. All authors read and approve the final manuscript.

\section{Acknowledgements}

We thank Birgit Ortel for her excellent technical assistance. The work was partially funded by the German Research Council (DFG, project HA2655/11-1 to B.H.).

\section{Author details}

${ }^{1}$ Department of Cell and Metabolic Biology, Leibniz Institute of Plant Biochemistry, Weinberg 3, Halle (Saale) 06120, Germany. ${ }^{2}$ Present address: Max Planck Institute for Chemical Ecology, Department of Biochemistry, Hans-Knoell-Str. 8, Jena 07745, Germany. ${ }^{3}$ Faculty of Chemistry and Mineralogy, Institute of Bioanalytical Chemistry, Centre for Biotechnology and Biomedicine, Leipzig University, Deutscher Platz 5, Leipzig 04103, Germany.

Received: 5 September 2012 Accepted: 12 November 2012 Published: 22 November 2012

\section{References}

1. Doares SH, Narvaez-Vasquez J, Conconi A, Ryan CA: Salicylic acid inhibits synthesis of proteinase inhibitors in tomato leaves induced by systemin and jasmonic acid. Plant Physiol 1995, 108:1741-1746.

2. Fonseca S, Chini A, Hamberg M, Adie B, Porzel A, Kramell R, et al: (+)-7-isoJasmonoyl-L-isoleucine is the endogenous bioactive jasmonate. Nat Chem Biol 2009, 5:344-350.

3. Pẽna-Cortés H, Sánchez-Serrano JJ, Mertens R, Willmitzer L, Prat S: Abscisic acid is involved in the wound-induced expression of the proteinase inhibitor II gene in potato and tomato. Proc Natl Acad Sci U S A 1989, 86:9851-9855.

4. Wasternack C, Stenzel I, Hause B, Hause G, Kutter C, Maucher H, et al: The wound response in tomato - role of jasmonic acid. J Plant Physiol 2006, 163:297-306.

5. Wasternack C: Jasmonates: an update on biosynthesis, signal transduction and action in plant stress response, growth and development. Ann Bot 2007, 100:681-697.

6. Koo AJK, Howe GA: The wound hormone jasmonate. Phytochemistry 2009, 70:1571-1580

7. Glauser G, Dubugnon L, Mousavi SA, Rudaz S, Wolfender JL, Farmer EE: Velocity estimates for signal propagation leading to systemic jasmonic acid accumulation in wounded arabidopsis. J Biol Chem 2009, 284:3450634513.

8. Koo AJK, Gao X, Jones AD, Howe GA: A rapid wound signal activates the systemic synthesis of bioactive jasmonates in arabidopsis. Plant J 2009, 59:974-986.

9. Glauser G, Grata E, Dubugnon L, Rudaz S, Farmer EE, Wolfender JL: Spatial and temporal dynamics of jasmonate synthesis and accumulation in arabidopsis in response to wounding. J Biol Chem 2008, 283:16400-16407.

10. Mueller MJ, Mène-Saffrané L, Grun C, Karg K, Farmer EE: Oxylipin analysis methods. Plant J 2006, 45:472-489.

11. Chehab EW, Kaspi R, Savchenko T, Rowe H, Negre-Zakharov F, Kliebenstein $D$, et al: Distinct roles of jasmonates and aldehydes in plant-defense responses. PLoS One 2008, 3:e1904.

12. Stenzel I, Hause B, Miersch O, Kurz T, Maucher $\mathrm{H}$, Weichert $\mathrm{H}$, et al: Jasmonate biosynthesis and the allene oxide cyclase family of Arabidopsis thaliana. Plant Mol Biol 2003, 51:895-911.

13. Taylor PJ: Matrix effects: the Achilles heel of quantitative highperformance liquid chromatography-electrospray-tandem mass spectrometry. Clin Biochem 2005, 38:328-334.

14. Tamogami S, Kodama O: Quantification of amino acid conjugates of jasmonic acid in rice leaves by high-performance liquid chromatography-turboionspray tandem mass spectrometry. $J$ Chromatogr A 1998, 822:310-315.

15. Ma Z, Ge L, Lee ASY, Yong JWH, Tan SN, Ong ES: Simultaneous analysis of different classes of phytohormones in coconut (Cocos nucifera L.) water using high-performance liquid chromatography and liquid chromatography-tandem mass spectrometry after solid-phase extraction. Anal Chim Acta 2008, 610:274-281.

16. Flores MI, Romero-Gonzalez R, Frenich AG, Vidal JL: QuEChERS-based extraction procedure for multifamily analysis of phytohormones in vegetables by UHPLC-MS/MS. J Sep Sci 2011, 34:1517-1524. 
17. Fan S, Wang X, Li P, Zhang Q, Zhang W: Simultaneous determination of 13 phytohormones in oilseed rape tissues by liquid chromatographyelectrospray tandem mass spectrometry and the evaluation of the matrix effect. J Sep Sci 2011, 34:640-650.

18. Durgbanshi A, Arbona V, Pozo O, Miersch O, Sancho JV, Gómez-Cadenas A: Simultaneous determination of multiple phytohormones in plant extracts by liquid chromatography-electrospray tandem mass spectrometry. J Agric Food Chem 2005, 53:8437-8442.

19. Forcat S, Bennett MH, Mansfield JW, Grant MR: A rapid and robust method for simultaneously measuring changes in the phytohormones ABA, JA and SA in plants following biotic and abiotic stress. Plant Meth 2008, 4:16-23.

20. Mannur VS, Patel D, Mastiholimath VS, Shah G: Selection of buffers in LCMS/MS: an overview. IJPSR 2011, 6:34-37.

21. Klimek-Turek A, Dzido TH: Separation selectivity of some phenolic acids in RP HPLC systems with binary mobile phase comprised various modifies. Adsorption 2010, 16:287-294.

22. Chen G, Zhang L-K, Pramanik BN: LC/MS:Theory, instrumentation, and applications to small molecules. Chapter 7. In HPLC for pharmaceutical scientists. Edited by Kazakevich Y, LoBrutto R.: John Willey \& Sons, inc. publication; 2007:281-347.

23. Fletcher AT, Mader JC: Hormone profiling by LC-QToF-MS/MS in dormant macadamia integrifolia: correlations with abnormal vertical growth. $J$ Plant Growth Regul 2007, 26:351-361.

24. Kallenbach M, Baldwin IT, Bonaventure G: A rapid and sensitive method for the simultaneous analysis of aliphatic and polar molecules containing free carboxyl groups in plant extracts by LC-MS/MS. Plant Meth 2009, 5:17-27.

25. Liu X, Yang YL, Lin WH, Tong JH, Huang ZG, Xiao LT: Determination of both jasmonic acid and methyl jasmonate in plant samples by liquid chromatography tandem mass spectrometry. Chin Sci Bull 2011, 55:2231-2235.

26. Frolov A, Hoffmann R: Separation of Amadori peptides from their unmodified analogs by ion-pairing RP-HPLC with heptafluorobutyric acid as ion-pair reagent. Anal Bioanal Chem 2008, 392:1209-1214.

27. López-Raéz JA, Verhage A, Fernandez I, Garcia JM, Azcon-Aguilar C, Flors V, et al: Hormonal and transcriptional profiles highlight common and differential host responses to arbuscular mycorrhizal fungi and the regulation of the oxylipin pathway. J Exp Bot 2010, 61:2589-2601.

28. Pan X, Welti R, Wang X: Simultaneous quantification of major phytohormones and related compounds in crude plant extracts by liquid chromatography-electrospray tandem mass spectrometry. Phytochemistry 2008, 69:1773-1781.

29. Chiwocha SDS, Abrams SR, Ambrose SJ, Cutler AJ, Loewen M, Ross ARS, et al: A method for profiling classes of plant hormones and their metabolites using liquid chromatography-electrospray ionization tandem mass spectrometry: an analysis of hormone regulation of thermodormancy of lettuce (Lactuca sativa L.) seeds. Plant J 2003, 35:405-417.

30. Miersch O, Wasternack C: Octadecanoid and jasmonate signaling in tomato (Lycopersicon esculentum mill.) leaves: endogenous jasmonates do not induce jasmonate biosynthesis. Biol Chem 2000, 381:715-722.

31. Suza WP, Rowe ML, Hamberg M, Staswick PE: A tomato enzyme synthesizes (+)-7-iso-jasmonoyl-L-isoleucine in wounded leaves. Planta 2010, 231:717-728

32. Novákova M, Motyka V, Dobrev PI, Malbeck J, Gaudinová A, Vanková R: Diurnal variation of cytokinin, auxin and abscisic acid levels in tobacco leaves. J Exp Bot 2005, 56:2877-2883.

33. Segarra G, Jáuregui $\mathrm{O}$, Casanova E, Trillas I: Simultaneous quantitative LCESI-MS/MS analyses of salicylic acid and jasmonic acid in crude extracts of Cucumis sativus under biotic stress. Phytochemistry 2006, 67:395-401.

34. Xiong XJ, Rao WB, Guo XF, Wang H, Zhang HS: Ultrasensitive determination of jasmonic acid in plant tissues using high-performance liquid chromatography with fluorescence detection. J Agric Food Chem 2012, 60:5107-5111.

35. Liu H, Li X, Xiao J, Wang S: A convenient method for simultaneous quantification of multiple phytohormones and metabolites: application in study of rice-bacterium interaction. Plant Meth 2012, 8:2.

36. MacNair JE, Lewis KC, Jorgenson JW: Ultrahigh-pressure reversed-phase liquid chromatography in packed capillary columns. Anal Chem 1997, 69:983-989.
37. Funk W, Dammann V, Donnevert G: Qualitätssicherung in der analytischen chemie: anwendungen in der umwelt-, lebensmittel- und werkstoffanalytik und medizintechnik. 2nd edition. Weinheim: Wiley-VCH Verlag GmbH \& Co; 2005.

38. Mielke K, Forner S, Kramell R, Conrad U, Hause B: Cell-specific visualization of jasmonates in wounded tomato and Arabidopsis leaves using jasmonate-specific antibodies. New Phytol 2011, 190:1069-1080.

39. Stenzel I, Hause B, Maucher H, Pitzschke A, Miersch O, Ziegler J, et al: Allene oxide cyclase dependence of the wound response and vascular bundlespecific generation of jasmonates in tomato - amplification in wound signalling. Plant J 2003, 33:577-589.

40. Landgraf R, Schaarschmidt S, Hause B: Repeated leaf wounding alters the colonization of Medicago truncatula roots by beneficial and pathogenic microorganisms. Plant Cell Environ 2012, 35:1344-1357.

41. Riemann M, Muller A, Korte A, Furuya M, Weiler EW, Nick P: Impaired induction of the jasmonate pathway in the rice mutant hebiba. Plant Physiol 2003, 133:1820-1830.

42. Staswick PE, Tiryaki I: The oxylipin signal jasmonic acid is activated by an enzyme that conjugates it to isoleucine in arabidopsis. Plant Cell 2004, 16:2117-2127.

43. Kitahara T, Iwamoto M, Takagi Y, Mori K, Matsui M: Synthesis of mediumcyclic and macro-cyclic compounds. 3. Synthesis of jasmine ketolactone. Agric Biol Chem 1984, 48:1731-1734.

44. Zimmerman DC, Feng P: Characterization of a prostaglandin-like metabolite of linolenic acid produced by a flaxseed extract. Lipids 1978, 13:313-316

45. Gómez-Cadenas A, Pozo OJ, García-Augustín P, Sancho JV: Direct analysis of abscisic acid in crude plant extracts by liquid chromatographyelectrospray/tandem mass spectrometry. Phytochem Anal 2002, 13:228-234

46. Miersch O, Schneider G, Sembdner G: Hydroxylated jasmonic acid and related compounds from Botryodiplodia theobromae. Phytochemistry 1991, 30:4049-4051.

47. Kramel R, Porzel A, Miersch O, Schneider G: Analysis of synthetic isoleucine conjugates of cucurbic acid isomers by liquid chromatography. Phytochem Anal 1999, 10:82-87.

doi:10.1186/1746-4811-8-47

Cite this article as: Balcke et al:: An UPLC-MS/MS method for highly sensitive high-throughput analysis of phytohormones in plant tissues. Plant Methods 2012 8:47.

\section{Submit your next manuscript to BioMed Central and take full advantage of:}

- Convenient online submission

- Thorough peer review

- No space constraints or color figure charges

- Immediate publication on acceptance

- Inclusion in PubMed, CAS, Scopus and Google Scholar

- Research which is freely available for redistribution 